(C2007 IEEE. Personal use of this material is permitted. However, permission to reprint/republish this material for advertising or promotional purposes or for creating new collective works for resale or redistribution to servers or lists, or to reuse any copyrighted component of this work in other works must be obtained from the IEEE. 


\section{Power Transformer Condition Assessment Using Oil UV - Spectrophotometry}

\author{
M. Arshad \\ Generation Engineering Maintenance Services \\ BC Hydro, 6911 Southpoint Drive, Burnaby \\ B.C. V3N 4X8, Canada
}

\author{
Syed M. Islam \\ Electrical \& Computer Engineering Department \\ Curtin University of Technology, Perth, \\ Western Australia
}

\begin{abstract}
Power transformers are considered critical equipment in an electric power transmission system. Electric utilities worldwide are now focusing on reliability centered maintenance of their assets for extended life and hence maximum return on investment. Recent utility survey shows that a large proportion of transformers has attained their designed life and is operating close to their nameplate rating or beyond. The load growth is compounding this problem on the existing transformers due to fewer extension projects, market deregulation and economic constraints. Diagnostics and proper monitoring plays key role in the life expectancy of a power transformer. Mineral oil in transformer is the inseparable component of the dielectric insulation system. The various contamination levels in oil can be examined and verified using UV-Spectrometry. The UV-spectrum analyzes the oil, qualitatively and quantitatively with respect to the contamination present in it and interprets dielectric response of the insulation system. The oil gets contaminated mainly due to the aging. Moisture, sludge, acids, metal particles and other compounds are produced due to aging insulation (mainly cellulose) which changes the oil chemical and physical properties. Accurate interpretation of the UV-Spectrophotometry and comparison with the past test signature provides sufficient assessment of power transformer insulation condition and predicts it's aging phenomena. This paper presents power transformer's dielectric response and aging assessment using oil contamination level identification with UV-Spectrometry measurements.
\end{abstract}

Index Terms-Power Transformer, Insulation Assessment, Aging, Oil, UV-Spectrophotometry, Condition Based Maintenance (CBM.

\section{INTRODUCTION}

A Power transformer is considered as most significant and vital element in the power delivery system. Due to the highly competitive electrical energy market it is required to enhance the system's reliability and availability with cost effectiveness. To maintain system reliability, its key components such as power transformers are required to undergo periodic diagnostics to avoid catastrophe and losses due to downtime [1]. The majority of the transformer fleet is serving close to or beyond its expected design life as majority of the transformers were installed soon after the World War II and in early 70's.
Due to increase in the peak load demand the hotspot temperature is rising high. The higher hotspot temperature forces sufficient reduction in asset's life and reliability. Due to elevated chances of sudden failure, long term revenue loss and high replacement cost is likely to happen. Therefore in the absence of transformer assessment, utilities may have significant concerns regarding reliability and performance of the system. Irrespective of the failure rate, transformers are of high importance from the point of view of asset management. System abnormalities such as loading, switching, ambient condition and poor maintenance normally contribute to accelerated aging. In the absence of critical components monitoring/diagnostics, the failure risk always remain high.

Insulation diagnostics is important to ascertain the overall integrity of the transformer to avoid any unscheduled outage, consequently restricting the financial/revenue losses along with environmental and collateral damages. It is always desired to anticipate the faults prior to become active in nature. With the application of online monitoring systems and diagnostics, accurate interpretations and timely decision incipient faults and accelerated deterioration of the insulation system can be better judged. Due to differences in operational criteria and climatic influence different characteristics and variety of problems subject to insulation deterioration and failure are observed in transformers.

The uv-spectrophotometry provides characteristics related to the contamination level in oil mainly due to the paper insulation (cellulose) which produces Furans, Acids, Water, Sludge and other compounds on its deterioration. Oil oxidation occurs due to the presence of oxygen and heat and may cause sludge formation. Aging is also strongly dependent on temperature, oxygen and water levels in the transformer. The transformer life can be maximized by controlling these variables. Metal particles are also found in the oil. Power transformer dielectric integrity highly depends upon the contamination level. Water and Furan are produced simultaneously and are the key indicators for the life expectancy of the power transformer. Continuing presence of $0.5 \%$ moisture in paper and $20 \mathrm{ppm}$ in oil are considered effective to cause sufficient dielectric degradation [2]. 
The solid insulation (cellulose) is the major element of the insulation system and its integrity is always required to be maintained in terms of its dielectric and tensile properties. The cellulose can be analyzed with the measure of the degree of polymerization (DP). The DP value below 300 is considered almost with no dielectric and mechanical properties and the transformer is not recommended for service where as the DP value above 1000 is considered with high dielectric and tensile properties [3].

Transformer failure statistics exhibit that most of the failures have occurred before reaching their rated life. Transformer failures due to dielectric problems are reported as high as $75 \%$ [4].

Due to the increase in the average age of the transformer population there is significant concern regarding the condition of its insulation system [5]. The average age of power transformer in Australia and New Zealand in 1995 was 28.6 years with considerable number $(65 \%)$ of transformers over 30 years [6][7] old. Transformers are considered of strategic importance in the network. The most frequent and immediate cause of the transformer costly and long-term outage is due to the winding insulation, on-load tap changer and bushings deterioration [8].

In the absence of insulation diagnostics, a good number of transformers failed before reaching their designed technical life. Average age that transformers have failed due to insulation deterioration during the last ten years was 17.8 years [9][10]. Therefore implementation of effective diagnostic tools and monitoring systems stands vital for early warning and assessment to avoid forced outage and catastrophic failure. This will allow for controlling the aging rate by suggesting new operational (loading) conditions and implementing the condition based maintenance strategies, extension in transformer life is achieved.

\section{UV SPECTROPHOTOMETRY}

UV-Spectrophotometry is an accurate and sensitive method to analyze impurities in transformer oil by using light absorbing properties of the sample. The light transmitted through oil sample containing (various contaminations) is decreased by that fraction being absorbed, detected and measured as a function of wavelength. A spectrophotometer measures the transmission, absorption or reflection of the light spectrum for the given wavelength.

The Beer's law provides a linear relationship between absorbance and concentration of an absorber of electromagnetic radiation such as:

$$
A=a_{\lambda} \times b \times c
$$

Where $A$ is the measured absorbance, $\lambda$ is a wavelengthdependent on absorption coefficient, $b$ is the path length, and $c$ is the sample concentration, also:

$$
A=\varepsilon_{\lambda} \times b \times c
$$

Where $\varepsilon_{\lambda}$ is the wavelength dependent on the molar absorption. The $\lambda$ subscript is often dropped with the understanding that a value for $\varepsilon$ is for a specific wavelength. If multiple species that absorb light at a given wavelength are present in a sample, the total absorbance at that wavelength is the sum due to all substances:

$$
A=\left(\varepsilon_{1} \times b \times c_{1}\right)+\left(\varepsilon_{2} \times b \times c_{2}\right)+\cdots \cdots \cdots \cdots
$$

The subscripts refer to the molar absorption and concentration of different absorbing impurities present in the sample. Experimental measurements are made in terms of transmittance $T$ which is defined as:

$$
T=\frac{P}{P_{o}}
$$

Where $P$ is the power of light after it passes through the sample and $P_{o}$ is the initial power of the light. The relation between $A$ and $T$ is defined as:

$$
A=-\log (T)=-\log \left(\frac{P}{P_{o}}\right)
$$

UV-Spectrophotometry is the non-intrusive test to determine the transformer integrity. The interpretation allows optimal maintenance procedure to achieve maximum practicable efficiency with enhanced reliability. The uvspectrophotometry provides reasonable information on the health of the power transformer to plan the cost effective maintenance, retirement, relocation and operational criteria. The spectrophotometry provides the over all assessment of the transformer with precision and the correlation between the transformer aging and uv trend can be easily established.

\section{EXPERIMENTAL RESULTS}

\section{A. Reflection Tests}

Reflection is the return of the radiation by a medium without a change in the wavelength. The reflection may be specular or diffuse in nature depending upon the angle of incidence with respect to angle of reflection. The glossier the surface, more specular is the reflection. Reflection is expressed in percentage $\left(\% R_{\lambda}\right)$ relative to the reflection from the standard substance such as:

$$
\% R_{\lambda}=\frac{S_{\gamma}-D_{\lambda}}{R_{\gamma}-D_{\lambda}} \times 100
$$


Where $S$ is the sample intensity at wavelength $\lambda, D$ is the dark intensity at wavelength $\lambda, R$ is the reference intensity at wavelength $\lambda$.

New transformer oil (mineral) uv-spectrophotometry for its reflection characteristics shows $90 \%$ reflection at the wavelength of $400 \mathrm{~nm}$ as shown in Figure 1. The reflection exhibits its characteristics in the wavelength range of 300 to $900 \mathrm{~nm}$. Above $80 \%$ reflection in fresh oil appears in the wavelength rang of 375 to $800 \mathrm{~nm}$.

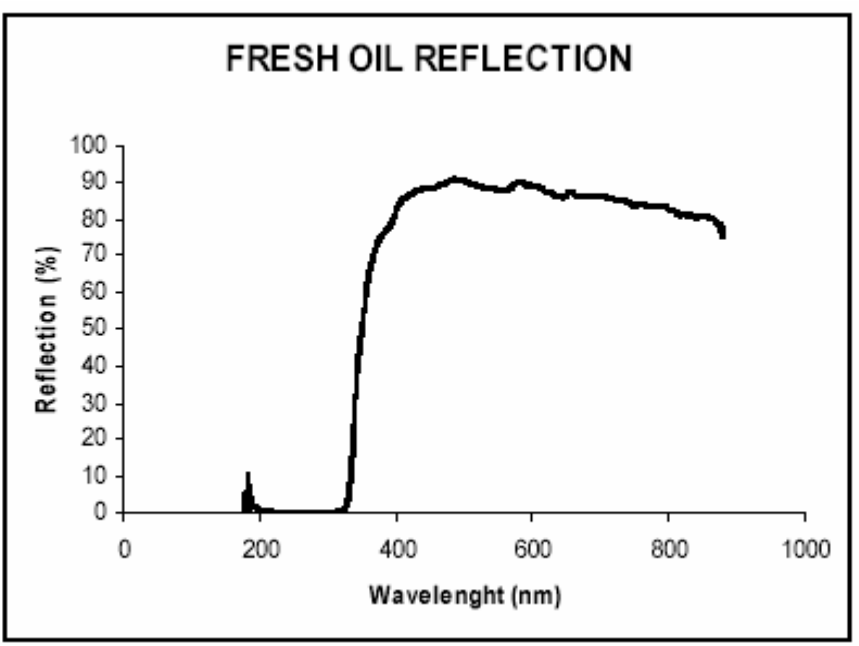

Figure 1: New Oil Reflection UV-Spectrophotometry Characteristics.

Five transformers rated at $10,15,15,10, \& 10$ MVA respectively were selected to perform the oil uvspectrophotometry tests as no oil was neither replaced or treated (regeneration) for any of these unit in their life time. The transformers were retired not due to specific problem but considered being very old. The service age was found to be $25,2422,22$ and 19 years respectively without any abnormality during life time. Also transformer oil sampled from a scrapped (burnt) 100 MVA transformer was obtained for reference. It is unwise to judge a transformer only on its service age with no diagnostics. The insulation may still be worth to withstand electrical and mechanical stresses and the asset may continue to serve for further period of time.

The uv-spectrophotometry for reflection exhibits a clear variation in the characteristics of various ages of the transformer oil as shown in Figure 2. In the wavelength spectrum from 300 to $900 \mathrm{~nm}$ the aging profile is prominent where as the scrapped transformer oil provide almost no reflection criteria in this range as shown in Figure 3. The deviation of the signature of aged oil form the new oil is significant in terms of wavelength spectrum and maximum percentage reflection as shown in Figure 2. The maximum reflection decreases with age as well as there is a shift in the wavelength for the maximum to occur.

The uv-spectrophotometry reflection characteristics of scrapped oil from completely burnt transformer appear only in the range of 177.5 to $189 \mathrm{~nm}$ wavelength spectrum. The $+25 \%$ $-60 \%$ reflection does not show any consistency in comparison to the above test characteristics as shown in Figure 3. The oil contains high carbon and water content including other contaminations.

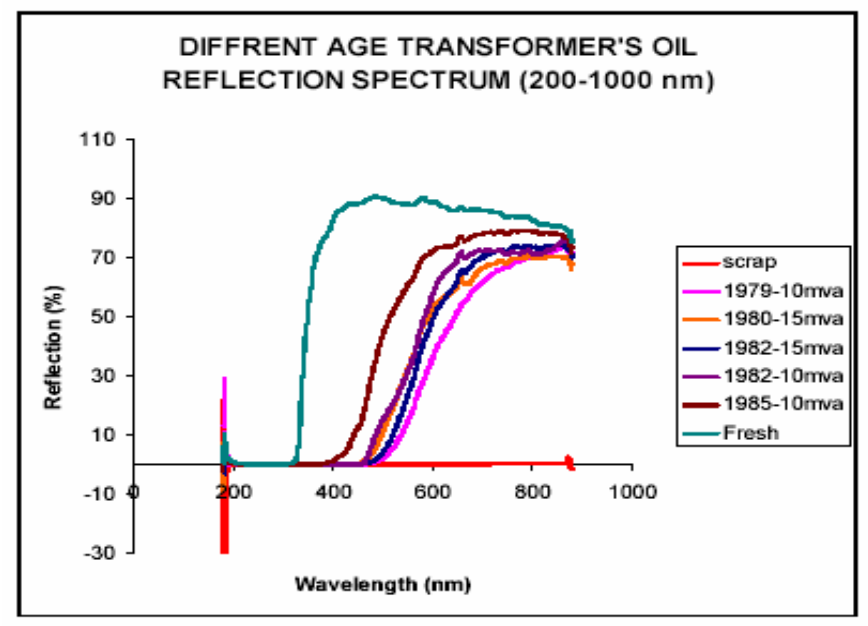

Figure 2: Different Age Transformer Oil Reflection UV Spectrum.

SCRAP TRANSFORMER OIL SPECULAR REFLECTION

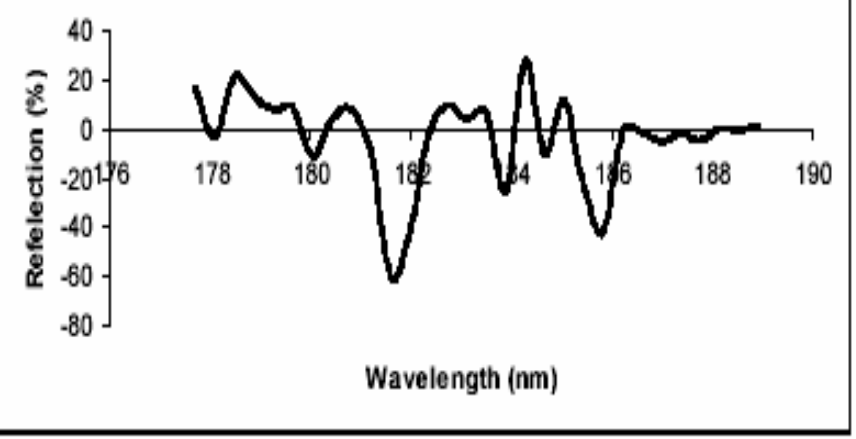

Figure 3: Scrap Transformer (burnt) Oil Reflection UV Characterisitcs

\section{B. Absorbance Tests}

The absorbance spectrum provides a measure of how much light is absorbed by the oil sample. The application is sensitive for the chemical concentrations in liquids and gaseous samples. The absorbance $A_{\lambda}$ is calculated as:

$A_{\lambda}=-\log _{10}\left(\frac{S_{\lambda}-D_{\lambda}}{R_{\lambda}-D_{\lambda}}\right)$

Where $S$ is the sample intensity at wavelength $\lambda, D$ is the dark intensity at wavelength $\lambda, R$ is the reference intensity at wavelength $\lambda$.

In confirmation to the reflection results the oil samples from the same units were tested for the absorbance uvspectrophotometry. The new transformer oil absorbance exhibits its characteristics between 175 and $350 \mathrm{~nm}$ 
wavelength uv-spectrum, with maximum absorbance at 250 $\mathrm{nm}$ wavelength as shown in Figure 4.

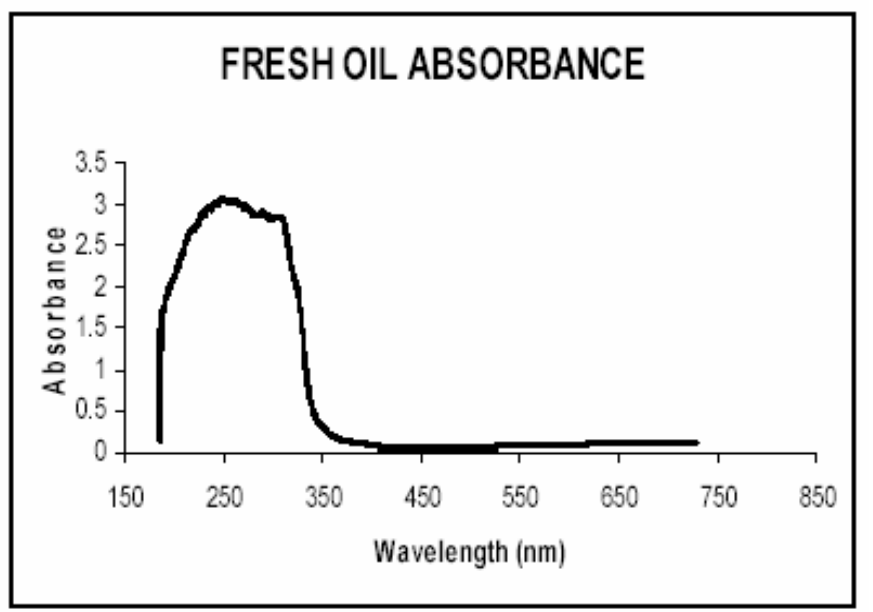

Figure 4: New Oil Absorbance UV Characteristics

The uv-spectrophotometry (absorbance) for the 10, 15, 15, $10, \& 10$ MVA transformers exhibits the respective characteristics in the range of 175-700 nm wavelength uvspectrum as demonstrated in Figure 5. The spectrographs represent that the absorbance increases with the oil deterioration and contaminations due to the aging properties of the insulation system (oil + cellulose). Also the bandwidth for the maximum absorbance increases and there is a wavelength shift as well with the age.

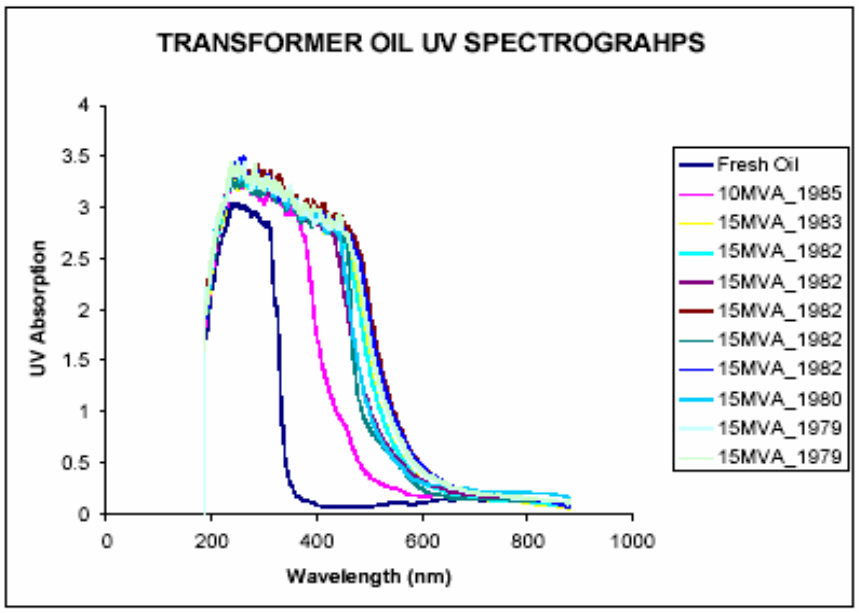

Figure 5: Different Age Transformer Oil Absorbance UV Spectrum

The absorbance spectrograph for the scrapped oil exhibits high absorbance level over the uv-spectrum from 200-900 nm wavelength as shown in Figure 6. Also there is a considerable noise from 200 to $540 \mathrm{~nm}$ range, mainly due to the variety of contamination including very high carbon and water contents.

The results demonstrated above points to the fact that strong correlation exists between the reflection/absorption indices and the level of contaminations present in the oil. Oil condition could be interpreted from scrapped to excellent.

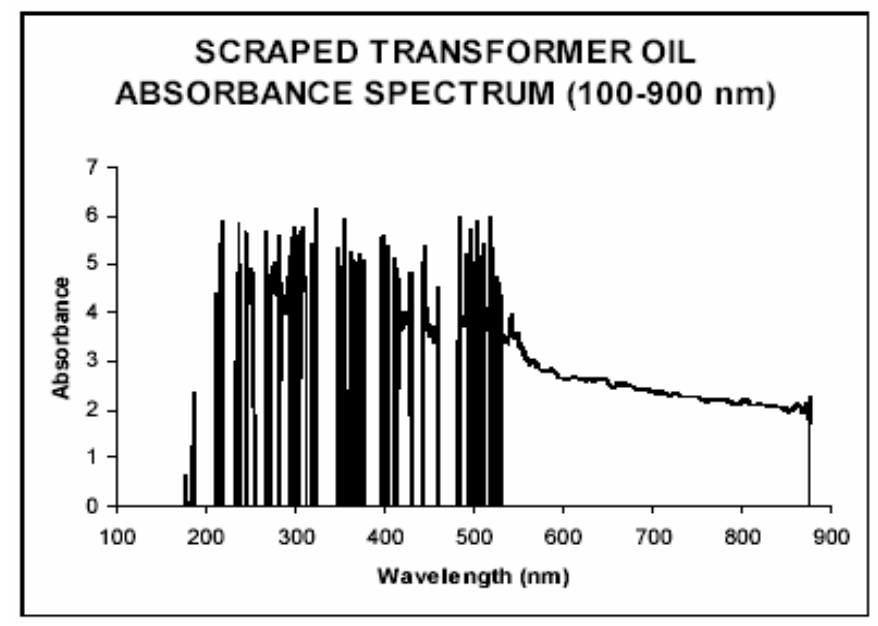

Figure 6: Scrap Transformer (burnt) Oil Absorbance UV Characterisitcs

\section{CONCLUSION}

UV-Spectrophotometry technique is proposed in this paper for detection of the level of contamination for oil regeneration/ replacement. The technique may prove to be an alternative to oil conductivity assessment. It also dictates the insulation system (oil + cellulose) condition due to normal as well as accelerated deterioration/ aging. Surprised failures and forced outages can be minimized effectively. A good return on the capital investment can be achieved as well as an extension in life is possible leading to proper asset management.

\section{REFERENCES}

[1] T. Strehl, E. Lemke, H. Elze, "On Line PD Measurement, Diagnostics Tools and Monitoring Strategy for Generators and Power Transformers," Workshop 2001, Alexandria, Virginia, December 3 \& 4, 2001

[2] S. M. Islam, T. Wu, G. Ledwich, "A Novel Fuzzy Logic Approach to Transformer Fault Diagnosis," IEEE Transaction on Dielectric and Electrical Insulation, Vol.7 No. 2,April 2000, pp. 177-186

[3] M. Arshad, S. M. Islam, A. Khaliq, "Power Transformer Aging and Life Extension," 8th International Conference on Probability Methods Applied to Power Systems, IEEE Iowa-Illinois (PMAPS 2004), Iowa State University Ames.

[4] W. H. Bartley, "Life Cycle Management of Utility Transformer Assets," Hartford Steam Boiler Inspection \& Insurance Company, Breakthrough Asset Management for the Restructured Power Industry forum, Salt Lake City, UT, October, 2002

[5] S. Tenbohlen, F. Figel, "Online Condition Monitoring for Power Transformers," IEEE Power Engineering Society Winter Meeting, Singapore January 2000.

[6] CIGRE Publication, Australia/ New Zealand Transformer Reliability Survey 1996 Report.

[7] F. Fetherston, B. Finlay, "Power Transformer Condition Assessment The Second Century and Beyond," AUPEC Conference September 2001.

[8] D. F. Peelo, et al, "A Value Based Methodology for Selecting On-line Condition Monitoring of Sub-station Power Equipment," EPRI Substation Equipment Diagnostics Conference V, New Orleans, Louisiana, $17^{\text {th }}$ Feb. 1997

[9] W. H. Bartley, "Analysis of Transformer Failures," Part 1 \& 2-1988 through 1997.

[10] M. Arshad, S. M. Islam, "Power transformer condition monitoring and assessment for strategic benefits," Australian Universities Power Engineering Conference (AUPEC' 2003). Christchurch New Zealand 